\title{
Structures of Schizosaccharomyces pombe Phosphofructokinase in the Presence of Either F6P or ATP
}

\author{
Shaun Benjamin*, Michael Radermacher*, Jörg Bär** and Teresa Ruiz* \\ * Dept. of Molecular Physiology and Biophysics, University of Vermont, Burlington, VT 05405 \\ ** Institut für Biochemie, Universität Leipzig, Liebigstr. 16, D-04103 Leipzig, Germany
}

Phosphofructokinase (Pfk-1), the main regulatory enzyme of the glycolytic pathway, catalyzes the phosphorylation of fructose 6-phosphate (F6P) in the presence of magnesium and adenosine triphosphate (ATP). The key role that the enzyme plays in regulation is evidenced by the large number of allosteric effectors (activators and inhibitors) that control its activity in eukaryotic organisms. Eukaryotic phosphofructokinases differ in size and oligomerization state, and they also exhibit a concentration dependent association-dissociation behavior that is exploited as a regulatory mechanism.

Saccharomyces cerevisiae Pfk-1 is the best characterized of the eukaryotic phosphofructokinase, both at the biochemical and structural level. S. cerevisiae Pfk-1 is an $835 \mathrm{kDa}$ heterooctamer which shows cooperative binding for F6P and non-cooperative binding for ATP. The three-dimensional structure of the F6P-bound state has been obtained by cryo-electron microscopy to $10.8 \AA$ resolution [1]. Later, this electron microscopy structure, in combination with molecular replacement using the bacterial enzyme, has provided the initial phases to solve the $\mathrm{x}$-ray structure of the F6P-bound state of the $12 \mathrm{~S}$ yeast truncatedtetramer [2]. The three-dimensional reconstruction from frozen-hydrated preparations of the ATP-bound state of Pfk-1 to $13 \AA$ resolution shows that the structure in the presence of ATP is more open. The calculated radius of gyration of $7.3 \mathrm{~nm}(7.0 \mathrm{~nm}$ for F6P) is in good agreement with SAXS data [3]. Additionally, there is a substantial decrease in the rotational angle between the top and bottom tetramer ( $75^{\circ}$ for the F6P structure and $46^{\circ}$ for the ATP structure), changes that have arisen from a reorientation of the subunits in the dimers. Schizosaccharomyces pombe has a singular Pfk-1 composed of eight identical subunits [4], a characteristic that brings it closer to the mammalian muscle enzymes.

We have carried out electron microscopy studies of the Pfk-1 from $S$. pombe in the presence of F6P and ATP to characterize the quaternary structure of the enzyme in the F6P-bound and ATP-bound states. Random conical tilt data sets were collected from deep stain preparations of the enzyme in each state. After correcting the contrast transfer functions for both, the un-tilted and tilted micrographs [5], the $0^{\circ}$ tilt images were separated into different classes. The classes were determined through several iterations of correspondence analysis combined with classification algorithms and multi-reference alignment. The tilted images were centered and three dimensional reconstructions were calculated from the members of each class using either back-projection [6] or fast Radon inversion algorithms [7,8].

S. pombe Pfk-1 in both the F6P-bound and the ATP-bound states can be described, similar to Pfk-1 from $S$. cerevisiae, as a dimer of tetramers and in projection, it shows two distinct subdomains: a diamond shaped headpiece which is connected by two well-defined densities (central connectors) to a more rectangular shaped basepiece. Our results confirm the presence of a variety of views of the particle, most of which can be interpreted as different views of the molecule rotating around its long axis. However, we have observed that this enzyme preparation shows higher variability than the $S$. cerevisiae Pfk- 1 . We are currently discerning if the angular difference between the tetramers in the F6P-bound and the ATP-bound state from $S$. pombe are similar to those of $S$. cerevisiae. An agreement in these values will indicate that the mechanism is similar in both hetero-octameric and homo-octameric enzymes.

\section{References:}

[1] T. Ruiz et al. (2003). Journal of Structural Biology. 143:124-134. 
[2] I. Mechin (2002). Ph. D. thesis dissertation.

[3] M. Laurent et al. (1984). Journal of Biological Chemistry 259, 3124-6.

[4] R. Reuter et al. (2000) Yeast 16:1273-1285. (2000)

[5] M. Radermacher et al. (2001). Journal of Structural Biology. 135, 26-37.

[6] M.Radermacher (1992) In: "Electron Tomography" (ed.J.Frank), Plenum Press, NY pp.91-115

[7] M. Radermacher (1997). Scanning Microscopy 11, 171-177.

[8] S. Lanzavecchia et al. (1999) Journal of Structural Biology. 128, 152-164.

[9] This work was supported by NIH R01 grant GM069551 (to T.R.) and has benefited from image analysis developments supported by NIH R01 grant GM068650 (to M.R.)
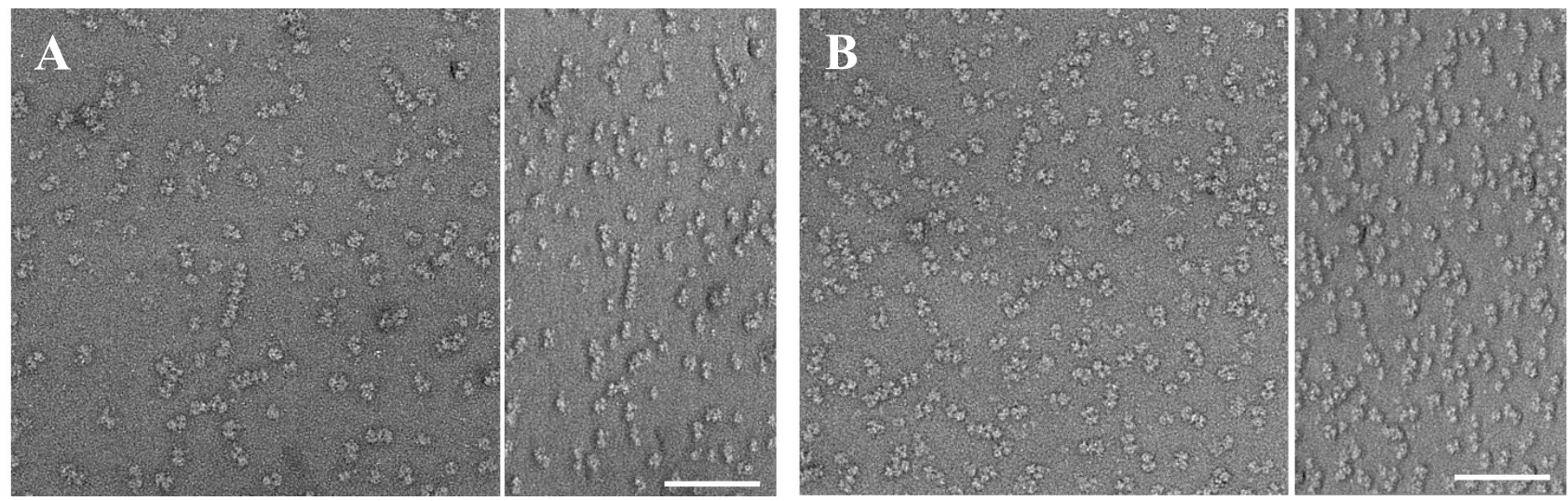

Figure 1. A) Tilt pair from F6P-bound state. Left: $0^{\circ}$-tilt micrograph. Right:55'-tilt of the same area. B)

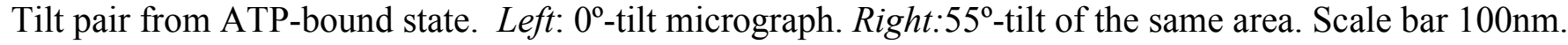

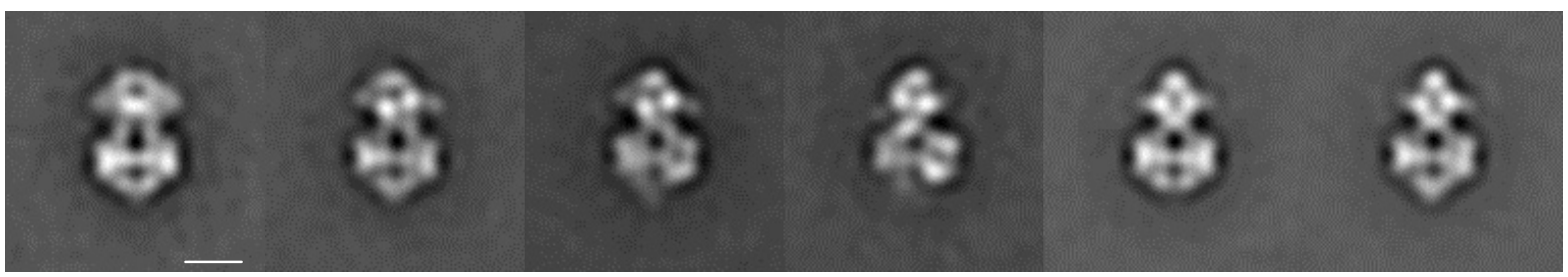

Figure 2. Class averages of the $0^{\circ}$-tilt images of the F6P-bound state after the final classification step. Scale bar $10 \mathrm{~nm}$
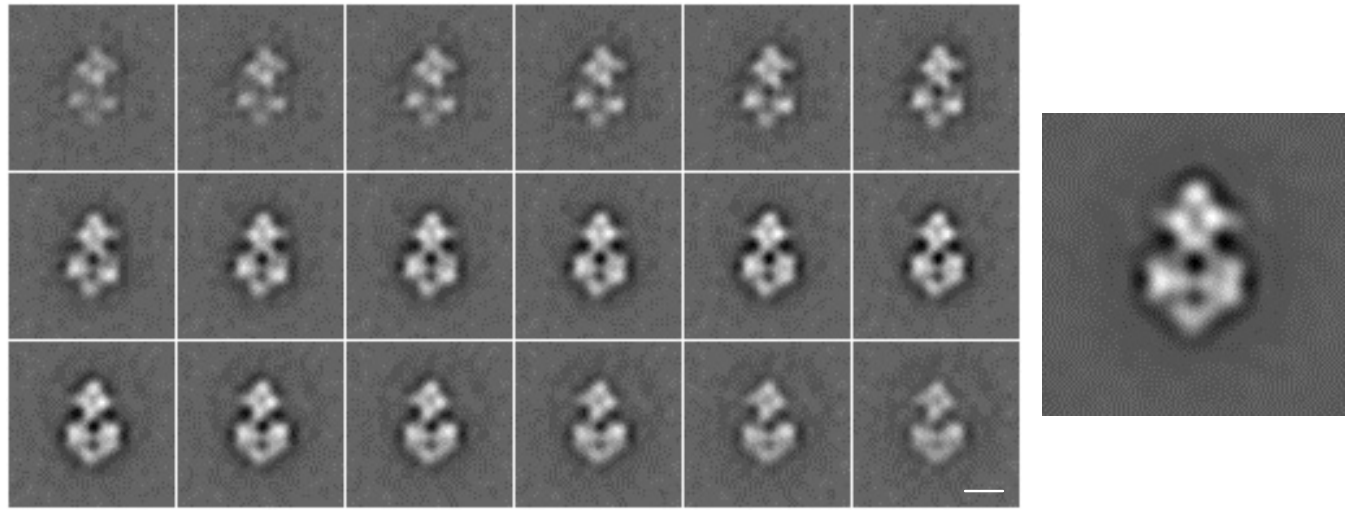

Figure 3. Slices through the 3D reconstruction of one class of the F6P-bound state. Scale bar 10nm 